\title{
Dispersion-cancelled and dispersion-sensitive quantum optical coherence tomography
}

\author{
Magued B. Nasr, Bahaa E. A. Saleh, Alexander V. Sergienko, and \\ Malvin C. Teich \\ Quantum Imaging Laboratory, Departments of Electrical \& Computer Engineering and \\ Physics, Boston University, Boston, MA 02215 \\ teich@bu.edu \\ http://www.bu.edu/qil
}

\begin{abstract}
Quantum optical coherence tomography (QOCT) makes use of an entangled twin-photon light source to carry out axial optical sectioning. We have probed the longitudinal structure of a sample comprising multiple surfaces in a dispersion-cancelled manner while simultaneously measuring the group-velocity dispersion of the interstitial media between the reflecting surfaces. The results of the QOCT experiments are compared with those obtained from conventional optical coherence tomography (OCT).
\end{abstract}

(C) 2004 Optical Society of America

OCIS codes: (110.4500) Optical coherence tomography; (170.4500) Optical coherence tomography; (270.0270) Quantum optics.

\section{References and links}

1. R. C. Youngquist, S. Carr, and D. E. N. Davies, "Optical coherence-domain reflectometry: a new optical evaluation technique," Opt. Lett. 12, 158-160 (1987).

2. K. Takada, I. Yokohama, K. Chida, and J. Noda, "New measurement system for fault location in optical waveguide devices based on an interferometric technique," Appl. Opt. 26, 1603-1606 (1987).

3. A. F. Fercher and E. Roth, "Ophthalmic laser interferometry," Proc. SPIE 658, 48-51 (1986).

4. D. Huang, E. A. Swanson, C. P. Lin, J. S. Schuman, W. G. Stinson, W. Chang, M. R. Hee, T. Flotte, K. Gregory, C. A. Puliafito, and J. G. Fujimoto, "Optical coherence tomography," Science 254, 1178-1181 (1991).

5. J. M. Schmitt, "Optical coherence tomography (OCT): A review," IEEE J. Sel. Topics Quantum Electron. 5, 1205-1215 (1999).

6. A. F. Fercher, W. Drexler, C. K. Hitzenberger, and T. Lasser, "Optical coherence tomography—principles and applications," Rep. Prog. Phys. 66, 239-303 (2003).

7. A. F. Abouraddy, M. B. Nasr, B. E. A. Saleh, A. V. Sergienko, and M. C. Teich, "Quantum-optical coherence tomography with dispersion cancellation," Phys. Rev. A 65, 053817 (2002).

8. M. B. Nasr, B. E. A. Saleh, A. V. Sergienko, and M. C. Teich, "Demonstration of dispersion-canceled quantumoptical coherence tomography," Phys. Rev. Lett. 91, 083601 (2003).

9. J. D. Franson, "Nonlocal cancellation of dispersion," Phys. Rev. A 45, 3126-3132 (1992).

10. A. M. Steinberg, P. G. Kwiat, and R. Y. Chiao, "Dispersion cancellation and high-resolution time measurements in a fourth-order optical interferometer," Phys. Rev. A 45, 6659-6665 (1992).

11. A. M. Steinberg, P. G. Kwiat, and R. Y. Chiao, "Dispersion cancellation in a measurement of the single-photon propagation velocity in glass," Phys. Rev. Lett. 68, 2421-2424 (1992).

12. T. S. Larchuk, M. C. Teich, and B. E. A. Saleh, "Nonlocal cancellation of dispersive broadening in Mach-Zehnder interferometers," Phys. Rev. A 52, 4145-4154 (1995).

13. L. Mandel and E. Wolf, Optical Coherence and Quantum Optics (Cambridge, New York, 1995), ch. 22.

14. C. K. Hong, Z. Y. Ou, and L. Mandel, "Measurement of subpicosecond time intervals between two photons by interference," Phys. Rev. Lett. 59, 2044-2046 (1987).

15. B. E. A. Saleh and M. C. Teich, Fundamentals of Photonics (Wiley, New York, 1991).

\#3987 - \$15.00 US

(C) 2004 OSA
Received 5 March 2004; revised 24 March 2004; accepted 25 March 2004

5 April 2004 / Vol. 12, No. 7 / OPTICS EXPRESS 1353 
16. A. F. Fercher, C. K. Hitzenberger, M. Sticker, R. Zawadzki, B. Karamata, and T. Lasser, "Dispersion compensation for optical coherence tomography depth-scan signals by a numerical technique," Opt. Commun. 204, 67-74 (2002).

17. M. Bass, Ed., Handbook of Optics, Vol. II, 2nd ed. (McGraw-Hill, New York, 1995), ch. 33, p. 67.

18. http://www.cvdmaterials.com/pdf/Zinc\%20Selenide.pdf

\section{Introduction}

Optical coherence tomography (OCT), a technique for carrying out axial sectioning of a specimen, has come into wide use [1]-[6]. A quantum version of OCT that makes use of an entangled twin-photon light source has recently been proposed [7] and experimentally demonstrated [8]. A particular merit of quantum optical coherence tomography (QOCT) is that it is inherently immune to group velocity dispersion (GVD) of the medium by virtue of the frequency entanglement associated with the twin-photon pairs [9]-[12]. Moreover, for sources of the same bandwidth, the entangled nature of the twin photons provides a factor of two enhancement in resolution relative to OCT.

In addition, QOCT permits us to directly determine the GVD coefficients of the interstitial media between the reflecting surfaces of the sample. A typical QOCT scan comprises two classes of features [7]. The features in the first class carry the information that is most often sought in OCT: the depth and reflectance of the surfaces that constitute the sample. Each of these features is associated with a reflection from a single surface and is immune to GVD. The features in the second class, in contrast, arise from cross interference between the reflection amplitudes associated with every pair of surfaces and are sensitive to the dispersion characteristics of the media between them. Measurement of the broadening of a feature associated with two consecutive surfaces directly yields the GVD coefficient of the interstitial medium lying between them. In an OCT scan, only a single class of features is observed; each feature is associated with the reflection from a single surface and is subject to the cumulative dispersion of the entire sample above it. Thus, GVD information is not directly accessible via OCT; to measure the GVD of a particular buried medium, one must consecutively compute the GVD of each of the constituent layers above it.

That said, the title of this paper is understood as follows: QOCT provides two classes of information, a dispersion-cancelled tomograph and a dispersion-sensitive determination of the GVD coefficients of the various media that comprise the sample.

In this letter we report a QOCT experiment in which we simultaneously probe the longitudinal structure of a layered sample and the GVD of the intervening media. A parallel experiment using conventional OCT, with a source of identical bandwidth, is conducted to provide a direct comparison of the two techniques. Our sample consisted of the four surfaces of two fused-silica windows sandwiching a 12-mm-thick $\mathrm{ZnSe}$ window (a highly dispersive material). Using the reflections from this sample, we observe an improvement in resolution of a factor of $\approx 5.5$. This improvement arises from the concatenation of two advantages inherent in QOCT: dispersion cancellation and the factor-of-two entanglement advantage. The GVD coefficient of $\mathrm{ZnSe}$ is simultaneously determined to be $\beta^{\prime \prime} \approx 4.0 \pm 0.8 \times 10^{-25} \mathrm{~s}^{2} \mathrm{~m}^{-1}$.

\section{Principles of QOCT}

We begin with a brief discussion of the principle underlying QOCT (for a comparative review of the theories of QOCT and OCT the reader is referred to Ref. [7]). A schematic of the QOCT arrangement is illustrated in Fig. 1. The entangled twin photons may be conveniently generated via spontaneous parametric downconversion (SPDC) [13]. In this process a monochromatic laser beam of angular frequency $\omega_{p}$, serving as the pump, is sent to a second-order nonlinear optical crystal (NLC). A fraction of the pump photons disintegrate into pairs of downcon- 
verted photons. Both downconveted photons have the same polarization and central angular frequency $\omega_{0}=\omega_{p} / 2$, corresponding to type-I degenerate SPDC. We direct our attention to a noncollinear configuration, in which the photons of the pairs are emitted in selected different directions (modes), denoted 1 and 2 in Fig. 1. Although each of the emitted photons has a broad spectrum in its own right, the sum of the angular frequencies must always equal $\omega_{p}$ by virtue of energy conservation.

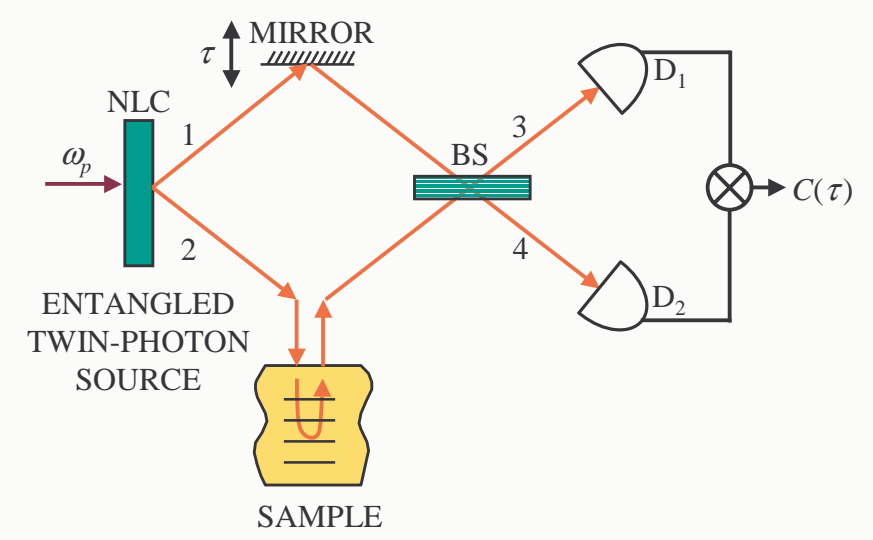

Fig. 1. Schematic of quantum optical coherence tomography (QOCT). A monochromatic laser of angular frequency $\omega_{p}$ pumps a nonlinear crystal (NLC), generating pairs of entangled photons. BS stands for beam splitter and $\tau$ is an adjustable temporal delay. $\mathrm{D}_{1}$ and $\mathrm{D}_{2}$ are single-photon-counting detectors that feed a coincidence circuit indicated by the symbol $\otimes$. The outcome of an experiment is the coincidence rate $C(\tau)$.

The twin-photon source is characterized by the frequency-entangled state

$$
|\psi\rangle=\int d \Omega \zeta(\Omega)\left|\omega_{0}+\Omega\right\rangle_{1}\left|\omega_{0}-\Omega\right\rangle_{2},
$$

where $\Omega$ is the angular-frequency deviation about the central angular frequency $\omega_{0}$ of the twin-photon wave packet, $\zeta(\Omega)$ is the spectral probability amplitude, and the power spectrum $S(\Omega)=|\zeta(\Omega)|^{2}$ is normalized such that $\int d \Omega S(\Omega)=1$. For simplicity we assume $S(\Omega)$ to be a symmetric function. Each photon of the pair resides in a single spatial mode, indicated by the subscripts 1 and 2 in Eq. (1).

The schematic illustrated in Fig. 1 has, at its heart, the two-photon interferometer considered by Hong, Ou, and Mandel (HOM) [14]. The conventional HOM configuration is modified by placing the sample to be probed in one arm and an adjustable temporal delay $\tau$ in the other arm. The entangled photons are directed to the two input ports of a symmetric beam splitter (BS). As shown in Fig. 1, beams 3 and 4 at its output ports are directed to two single-photon-counting detectors, $D_{1}$ and $D_{2}$, respectively. The coincidence rate of photons arriving at the two detectors, $C(\tau)$, is recorded within a time window determined by a coincidence circuit (indicated by $\otimes$ ).

An experiment is conducted by sweeping the temporal delay $\tau$ and recording the interferogram $C(\tau)$. If a mirror were to replace the sample, this would trace out a dip in the coincidence rate whose minimum would occur when arms 1 and 2 of the interferometer had equal path lengths. This dip would result from interference of the two photon-pair probability amplitudes, viz. reflection or transmission of both photons at the beam splitter. 
A weakly reflecting sample is described by a transfer function $H(\omega)$, characterizing the overall reflection from all structures that comprise the sample, at angular frequency $\omega$ :

$$
H(\omega)=\int_{0}^{\infty} d z r(z, \omega) e^{i 2 \phi(z, \omega)} .
$$

The quantity $r(z, \omega)$ is the complex reflection coefficient from depth $z$ and $2 \phi(z, \omega)$ is the round-trip phase accumulated by the wave while travelling through the sample to depth $z$. Losses are not included in this expression although they are accounted for subsequently. As shown previously [7], the coincidence rate $C(\tau)$ is then given by

$$
C(\tau) \propto \Lambda_{0}-\operatorname{Re}\{\Lambda(2 \tau)\}
$$

where

$$
\Lambda_{0}=\int d \Omega\left|H\left(\omega_{0}+\Omega\right)\right|^{2} S(\Omega)
$$

and

$$
\Lambda(\tau)=\int d \Omega H\left(\omega_{0}+\Omega\right) H^{*}\left(\omega_{0}-\Omega\right) S(\Omega) e^{-i \Omega \tau}
$$

represent the constant and varying contributions, respectively. The interferogram $C(\tau)$ yields useful information about the transfer function $H(\omega)$ and hence about the reflectance $r(z, \omega)$ [7].

\section{Experimental arrangement}

The details of the QOCT experimental arrangement are shown in Fig. 2. For QOCT scans, the dotted components (mirrors $\mathrm{M}_{1}$ and $\mathrm{M}_{2}$, as well as detector $\mathrm{D}_{3}$ ) are removed. The entangled photons, centered about $\lambda_{0}=812 \mathrm{~nm}$ and emitted in a non-collinear configuration, travel in beams 1 and 2 . The photon in beam 1 travels through a temporal delay $\tau$ before it enters the input port of the first beam splitter, $\mathrm{BS}_{1}$. The second photon in beam 2 goes through a second beam splitter, $\mathrm{BS}_{2}$, which ensures normal incidence onto the sample. The photon returned from the sample is directed to the other input port of $\mathrm{BS}_{1}$. Beams 3 and 4 , at the output of $\mathrm{BS}_{1}$, are directed to detectors $\mathrm{D}_{1}$ and $\mathrm{D}_{2}$, respectively.

For OCT scans, the photons in beam 1 are discarded and mirrors $M_{1}$ and $M_{2}$ remain in place (see Fig. 2). The photons in beam 2 then simply serve as a short-coherence-time light source. The reflections from the sample and mirror $\mathrm{M}_{1}$, after recombination at beam splitter $\mathrm{BS}_{2}$ are directed to detector $\mathrm{D}_{3}$ via mirror $\mathrm{M}_{2}$. The result is a simple Michelson interferometer, the standard configuration for OCT. To conduct an experiment, mirror $\mathrm{M}_{1}$ is scanned thereby sweeping the delay $\tau$ at the bottom of Fig. 2, and the singles rate is recorded, forming the OCT interferogram $I(\tau)$. This arrangement permits a fair comparison between QOCT and OCT since both make use of a light source with identical spectrum. An alternative configuration for OCT scans, which we sometimes use, involves eliminating $\mathrm{M}_{2}$ and $\mathrm{D}_{3}$ altogether and simply recording the singles rate $I(\tau)$ at $\mathrm{D}_{1}$ or $\mathrm{D}_{2}$.

\section{Results for a non-dispersive sample}

The first experiment makes use of a four-surface sample $(j=1, \ldots, 4)$ comprising two thin fused-silica (FS) windows separated by air, as illustrated at the very top of Fig. 3. The transfer function of the sample, $H(\omega)$, is therefore given by

$$
H(\omega)=H_{\mathrm{F}}(\omega)+H_{\mathrm{B}}(\omega) e^{i \omega \tau_{d}} e^{i \omega \tau_{D}},
$$

where $H_{\mathrm{F}}(\omega)=r_{1}+r_{2} e^{i \omega \tau_{d}}$ and $H_{\mathrm{B}}(\omega)=r_{3}+r_{4} e^{i \omega \tau_{d}}$ are the transfer functions of the front (F) and back (B) fused-silica windows, respectively. The round-trip time $\tau_{d}$ between the two

\#3987 - \$15.00 US

(C) 2004 OSA 


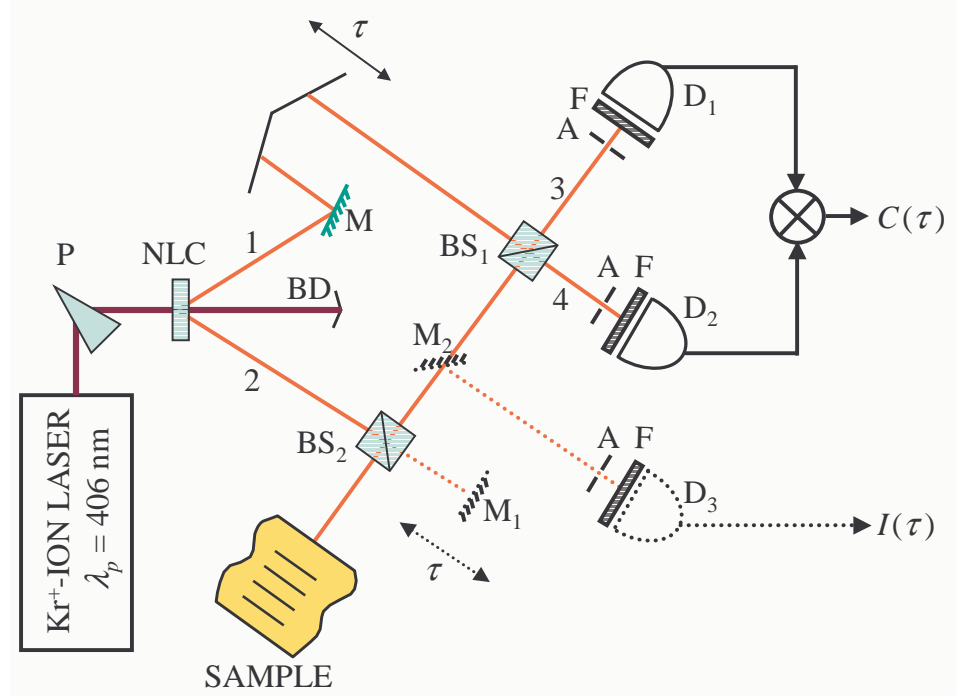

Fig. 2. Experimental arrangement for quantum/classical optical coherence tomography (QOCT/OCT). A monochromatic $\mathrm{Kr}^{+}$-ion laser operated at $\lambda_{p}=406 \mathrm{~nm}$ pumps an 8mm-thick type-I $\mathrm{LiIO}_{3}$ nonlinear crystal (NLC) after passage through a prism, $\mathrm{P}$, and an aperture (not shown), which remove the spontaneous glow of the laser tube. BD stands for beam dump (to block the pump), BS for beam splitter, $\mathrm{M}$ for mirror, A for 2.2-mm aperture, $\mathrm{F}$ for long-pass filter with cutoff at $725 \mathrm{~nm}$, and D for single-photon-counting detector (EG\&G, SPCM-AQR-15). The quantity $\tau$ represents a temporal delay. For QOCT scans, the dotted components $\mathrm{M}_{1}, \mathrm{M}_{2}$ and $\mathrm{D}_{3}$ are removed, the delay $\tau$ at the top of the figure is swept, and the coincidence rate $C(\tau)$ is measured within a 3.5-nsec time window. For OCT scans, beam 1 is discarded (beam 2 serves as the short-coherence-time light source), mirror $\mathrm{M}_{1}$ is scanned thereby sweeping the delay $\tau$ at the bottom of the figure, and the singles rate $I(\tau)$ is recorded.

surfaces of each fused-silica window is given by $\tau_{d}=2 n d / c$, whereas $\tau_{D}=2 D / c$ is the time delay associated with a round trip through the intervening air. The reflectance from each surface of each window is $\left|r_{j}\right|^{2}=0.04$ at normal incidence; each window has a thickness $d=90 \mu \mathrm{m}$ (which is greater than the $37-\mu \mathrm{m}$ coherence length of the source); $D=4.5 \mathrm{~mm}$ is the air spacing between the two windows; $c$ is the speed of light in vacuum; and $n \approx 1.5$ is the refractive index of the fused silica (which is taken to be independent of $\omega$ by virtue of the low dispersiveness of this material). Under these conditions, Eqs. (4)-(6) yield

$$
\Lambda_{0}=\sum_{j=1}^{4}\left|r_{j}\right|^{2}
$$

and

$$
\Lambda(\tau)=\Lambda_{\mathrm{F}}(\tau)+\Lambda_{\mathrm{B}}\left(\tau-2 \tau_{0}\right)+\Lambda_{\mathrm{FB}}\left(\tau-\tau_{0}\right),
$$

where $\tau_{0} \equiv \tau_{d}+\tau_{D}$.

The first two terms in Eq. (8), $\Lambda_{\mathrm{F}}(\tau)$ and $\Lambda_{\mathrm{B}}(\tau)$, represent the individual QOCT scans [8] of the front $(\mathrm{F})$ and back (B) fused-silica windows, respectively, and are given by

$$
\begin{aligned}
\Lambda_{\mathrm{F}}(\tau)= & \left|r_{1}\right|^{2} s(\tau)+\left|r_{2}\right|^{2} s\left(\tau-2 \tau_{d}\right) \\
& +2 \operatorname{Re}\left\{r_{1} r_{2}^{*} s\left(\tau-\tau_{d}\right) e^{i \omega_{0} \tau_{d}}\right\},
\end{aligned}
$$




$$
\begin{aligned}
\Lambda_{\mathrm{B}}(\tau)= & \left|r_{3}\right|^{2} s(\tau)+\left|r_{4}\right|^{2} s\left(\tau-2 \tau_{d}\right) \\
& +2 \operatorname{Re}\left\{r_{3} r_{4}^{*} s\left(\tau-\tau_{d}\right) e^{i \omega_{0} \tau_{d}}\right\},
\end{aligned}
$$

where $s(\tau)$ is the Fourier transform of the power spectrum $S(\Omega)$. The QOCT scan of the front window $\left(\Lambda_{\mathrm{F}}\right)$ and the back window $\left(\Lambda_{\mathrm{B}}\right)$ each comprise three terms. The first two of these are dips that arise from reflections from the front and back surfaces of each window $\left(r_{1}\right.$ and $r_{2}$ for the front window; $r_{3}$ and $r_{4}$ for the back window). These dips, each of which is associated with

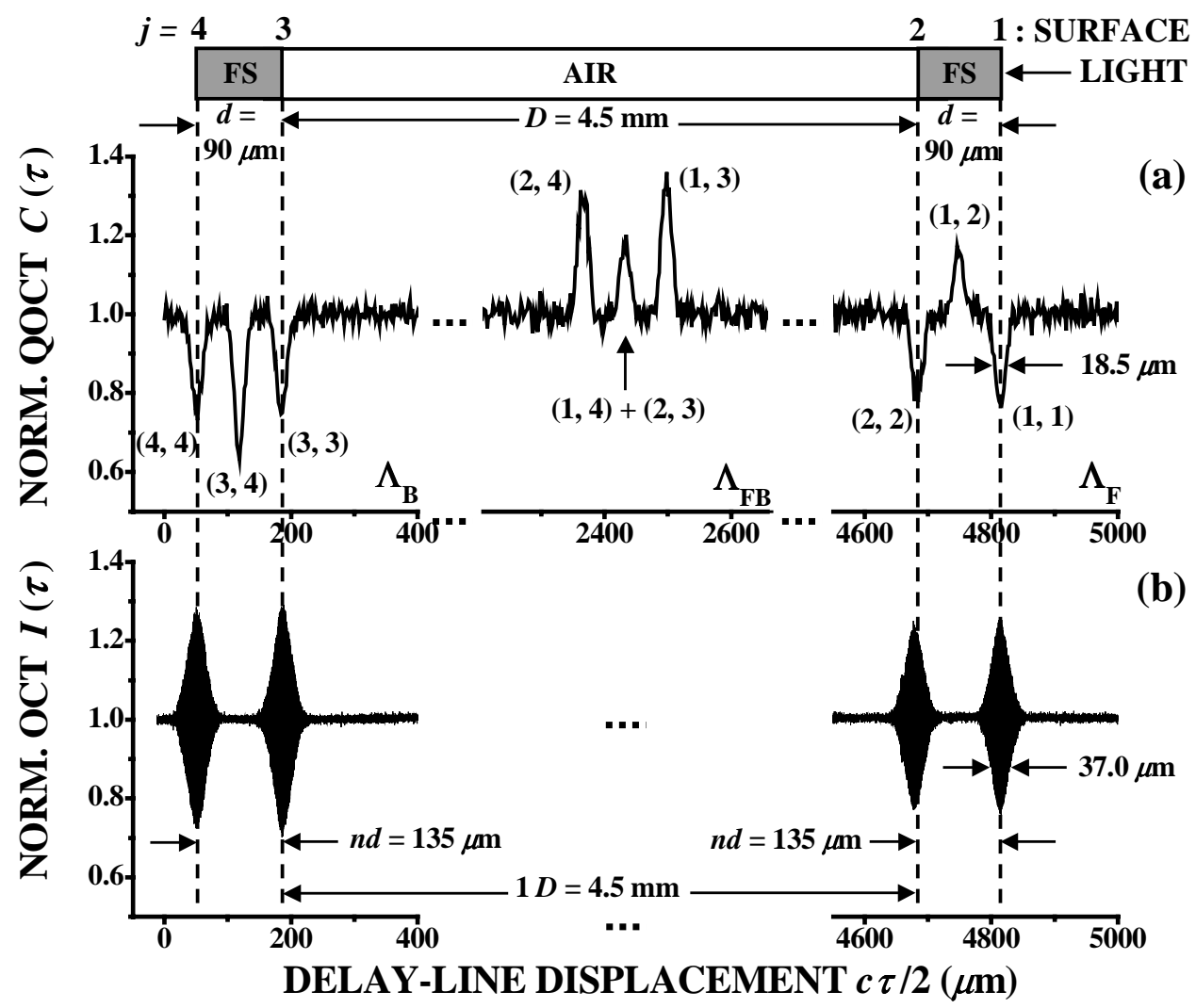

Fig. 3. QOCT and OCT normalized interferograms for two $d=90-\mu \mathrm{m}$ fused-silica (FS) windows separated by $D=4.5 \mathrm{~mm}$ of air. The four surfaces that comprise the sample are numbered, as shown at the top of the figure. The abscissa is the scaled temporal delay $c \tau / 2$, which represents the displacement of the delay line for both experiments (OCT and QOCT). (a) Coincidence rate $C(\tau)$ normalized to the constant background $\Lambda_{0}$ (the normalized QOCT interferogram). Features labelled $(j, j)$ correspond to reflections from the $j^{\text {th }}$ surface whereas those labelled $(j, k), j \neq k$, correspond to cross-interference between pairs of surfaces. The outermost clusters of features, labelled $\Lambda_{\mathrm{F}}$ and $\Lambda_{\mathrm{B}}$, correspond to the triplets of terms appearing in Eq. (9). The center cluster, labelled $\Lambda_{\mathrm{FB}}$, corresponds to the terms appearing in Eq. $(10)$, in which $(1,4)$ and $(2,3)$ overlap. The power of the pump laser was $7 \mathrm{~mW}$, which resulted in a power in each of the downconverted beams of $43 \mathrm{pW}$. (b) Singles rate $I(\tau)$ normalized to constant background (the normalized OCT interferogram). The power of the pump laser was $13 \mathrm{~mW}$, resulting in a downconverted-beam power of 80 pW. 
the reflection from a single surface, carry the information about the structure of the sample that is often sought in OCT. They are separated by $\tau_{d}$ and are expected to exhibit $25 \%$ visibility by virtue of Eqs. (3), (7), (8), and (9), since $\left|r_{j}\right|^{2}=0.04$ for $j=1, \ldots, 4$. The third term in $\Lambda_{\mathrm{F}}$ or $\Lambda_{\mathrm{B}}$ [see Eq. (9)], which appears midway between the two dips, arises from cross interference between the reflection amplitudes associated with the two surfaces. This intermediate term ranges from a peak to a dip depending on the values of $\omega_{p}, n, d$, the arguments of $r_{1}$ and $r_{2}$ (front window), and the arguments of $r_{3}$ and $r_{4}$ (back window).

The third term in Eq. (8), $\Lambda_{\mathrm{FB}}(\tau)$, which is given by

$$
\begin{aligned}
\Lambda_{\mathrm{FB}}(\tau)=2 \operatorname{Re}\left\{\left[r_{1} r_{3}^{*} s(\tau)\right.\right. & +\left(r_{1} r_{4}^{*} e^{-i \omega_{0} \tau_{d}}+r_{2} r_{3}^{*} e^{i \omega_{0} \tau_{d}}\right) s\left(\tau-\tau_{d}\right) \\
& \left.\left.+r_{2} r_{4}^{*} s\left(\tau-2 \tau_{d}\right)\right] e^{i \omega_{0} \tau_{0}}\right\},
\end{aligned}
$$

arises from cross interference between the reflection amplitudes associated with pairs of surfaces (one from each window) when the two windows are incorporated in a sample. These cross-window intermediate terms are of the same nature as the third terms in $\Lambda_{\mathrm{F}}$ and $\Lambda_{\mathrm{B}}$ for the individual QOCT scans of each window [see Eq. (9)]. Substituting Eqs. (7)-(10) into Eq. (3) yields an interferogram that contains ten varying terms.

The experimental QOCT interferogram for this sample, normalized to the constant background $\Lambda_{0}$, is plotted in Fig. 3(a). The dips associated with the reflection from single surfaces are labelled $(j, j)$, where $j$ is the surface number as shown at the top of the figure. These dips are separated by the optical path length of the medium between them: going from right to left in the figure, the values of the optical path lengths are $n d=135 \mu \mathrm{m}$ (front window), $n_{\text {air }} D=1 D=4.5$ $\mathrm{mm}$ (intervening air), and $n d=135 \mu \mathrm{m}$ (back window). All $(j, j)$ dips exhibit $23 \%$ visibility, in close agreement with the theoretically expected value of $25 \%$.

The cross-interference features associated with reflections from pairs of surfaces are labelled $(j, k), j \neq k$. For example, in the individual QOCT scan of the front window, $\Lambda_{\mathrm{F}}(\tau)$ in Eq. (9), the feature corresponding to the term $2 \operatorname{Re}\left\{r_{1} r_{2}^{*} s\left(\tau-\tau_{d}\right) e^{i \omega_{0} \tau_{d}}\right\}$ is labelled $(1,2)$. Because of the low dispersion of the layers comprising this sample, all dips and peaks in the plot have the same width $(18.5 \mu \mathrm{m}$ full width at half maximum, FWHM). It is of interest to note that although the two fused-silica windows are identical, the intermediate feature of the front window, labelled $(1,2)$, turns out to be a peak while that of the back window, labelled $(3,4)$, is a dip. This can result from a slight thickness mismatch, of the order of the wavelength of the light, between the two windows, as mentioned earlier. The abscissa is represented in units of the scaled temporal delay $c \tau / 2$, representing the physical displacement of the delay line.

The OCT interferogram for the same sample is expected to consist of four interferencefringe envelopes, each with visibility calculated to be $30 \%$, separated by the same optical path length as the $(j, j)$ dips. The experimental OCT interferogram for this sample, normalized to the constant background, is displayed in Fig. 3(b). The centers of the envelopes exhibit 26\% visibility, which is close to the expected value.

It is apparent that the $18.5-\mu \mathrm{m}$ FWHM of the dips observed in QOCT provides a factor of 2 improvement in resolution over the 37- $\mu \mathrm{m}$ FWHM of the envelopes observed in OCT. This improvement, which is in accord with theory [7], ultimately results from the entanglement inherent in the nonclassical light source used in QOCT [8].

\section{Results for a dispersive sample}

To demonstrate the dispersion-cancellation capability of QOCT, as well as the ability to measure GVD coefficients, we carry out a second QOCT/OCT experiment with a highly dispersive medium: a 12-mm-thick window of ZnSe placed between the two fused-silica windows. The $\mathrm{ZnSe}$ window is slightly canted with respect to the incident beam to divert back-reflections, as schematized at the top of Fig. 4.

\#3987 - \$15.00 US

(C) 2004 OSA
Received 5 March 2004; revised 24 March 2004; accepted 25 March 2004 5 April 2004 / Vol. 12, No. 7 / OPTICS EXPRESS 1359 


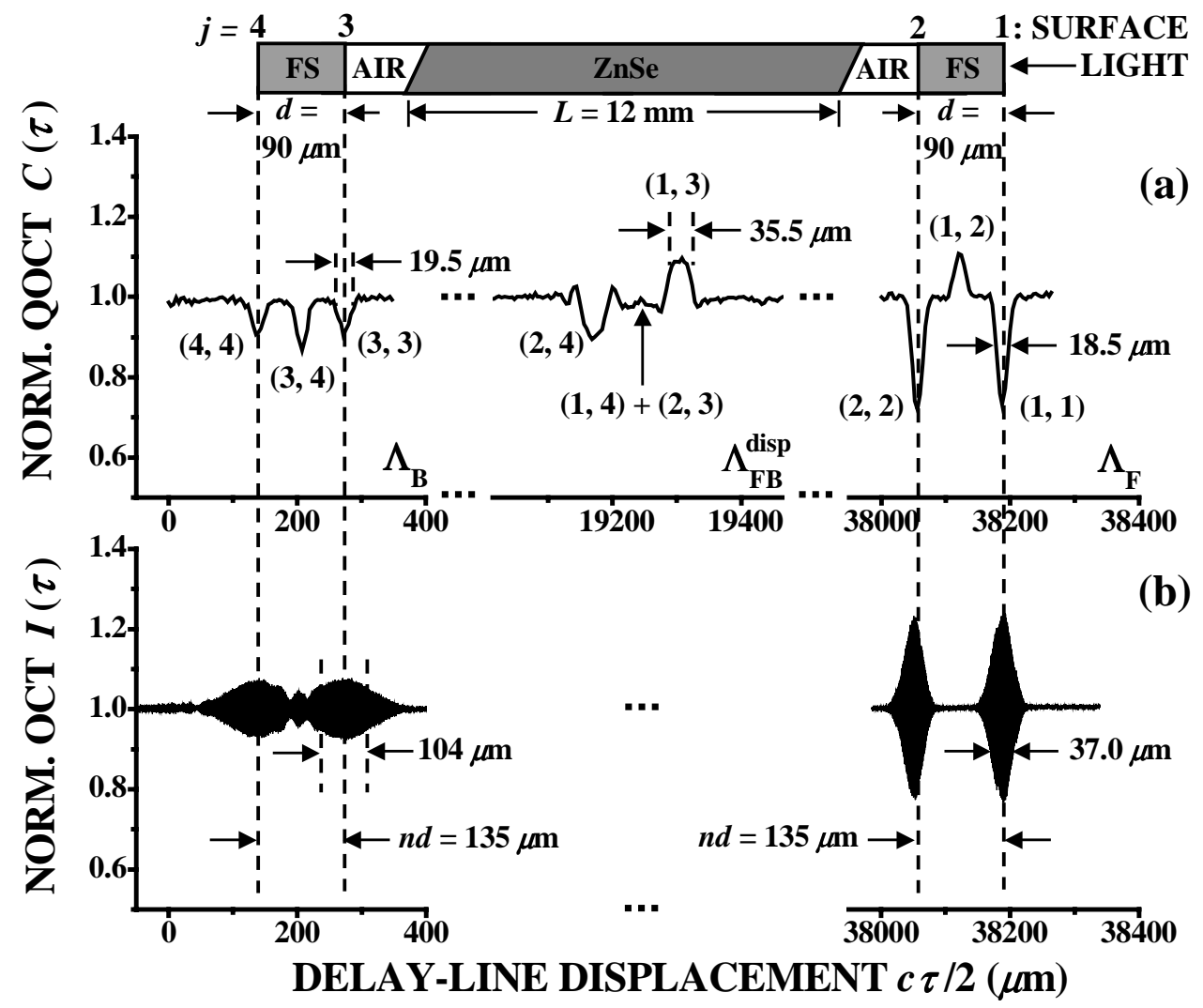

Fig. 4. QOCT and OCT normalized interferograms for two $d=90-\mu \mathrm{m}$ fused-silica (FS) windows sandwiching an $L=12$-mm window of highly dispersive $\mathrm{ZnSe}$. As shown at the top of the figure, the $\mathrm{ZnSe}$ is slightly canted with respect to the incident beam (arrow) to divert back-reflections. The four surfaces that comprise the sample are numbered, as shown at the top of the figure. The abscissa is the scaled temporal delay $c \tau / 2$, which represents displacement of the delay line for both experiments (OCT and QOCT). (a) Coincidence rate $C(\tau)$ normalized to the constant background $\Lambda_{0}$ (the QOCT normalized interferogram). Features labelled $(j, j)$ correspond to reflections from the $j^{\text {th }}$ surface whereas those labelled $(j, k), j \neq k$, correspond to cross-interference between pairs of surfaces. The outermost clusters of features, labelled $\Lambda_{\mathrm{F}}$ and $\Lambda_{\mathrm{B}}$, correspond to the features appearing in the first and second terms of Eq. (13), respectively. The center cluster, labelled $\Lambda_{\mathrm{FB}}^{\text {disp }}$, corresponds to the terms appearing in Eq. (14), in which $(1,4)$ and $(2,3)$ overlap. The power of the pump laser was $120 \mathrm{~mW}$, which resulted in a power in each of the downconverted beams of $685 \mathrm{pW}$. (b) Singles rate $I(\tau)$ normalized to constant background (the normalized OCT interferogram). The power of the pump laser was $120 \mathrm{~mW}$, resulting in a downconvertedbeam power of $685 \mathrm{pW}$.

The transfer function of this composite sample is

$$
H^{\operatorname{disp}}(\omega)=H_{\mathrm{F}}(\omega)+\alpha H_{\mathrm{B}}(\omega) e^{i \omega \tau_{d}} e^{i 2 \beta(\omega) L},
$$

where $\beta(\omega)$ is the wave number and $L=12 \mathrm{~mm}$ is the thickness of the $\mathrm{ZnSe}$ window. We have ignored the air gaps between the fused-silica and $\mathrm{ZnSe}$ windows since this has no material 
effect on the results. The factor $\alpha$ represents the loss introduced by the ZnSe window; it is measured to be $|\alpha|^{2}=0.32$. The phase factor $e^{i 2 \beta(\omega) L}$ in Eq. (11), which represents the roundtrip propagation through the dispersive medium, replaces the phase factor $e^{i \omega \tau_{D}}$ in Eq. (6), acquired through propagation in air. We now carry out the usual Taylor expansion of $\beta\left(\omega_{0}+\Omega\right)$ to second order in $\Omega$, where $\Omega$ is the angular frequency deviation about $\omega_{o}: \beta\left(\omega_{0}+\Omega\right) \approx$ $\beta_{0}+\beta^{\prime} \Omega+\beta^{\prime \prime} \Omega^{2}$, where $\beta^{\prime}$ is the inverse of the group velocity at $\omega_{0}$, and $\beta^{\prime \prime}$ represents groupvelocity dispersion (GVD) [15].

Substituting $H^{\text {disp }}(\omega)$ from Eq. (11) into Eqs. (4) and (5) yields

$$
\Lambda_{0}=\sum_{j=1}^{2}\left|r_{j}\right|^{2}+|\alpha|^{2} \sum_{j=3}^{4}\left|r_{j}\right|^{2}
$$

and

$$
\Lambda(\tau)=\Lambda_{\mathrm{F}}(\tau)+|\alpha|^{2} \Lambda_{\mathrm{B}}\left(\tau-2 \tau_{1}\right)+\Lambda_{\mathrm{FB}}^{\mathrm{disp}}\left(\tau-\tau_{1}\right),
$$

respectively, where $\tau_{1} \equiv \tau_{d}+\tau_{L}$, and $\tau_{L}=2 \beta^{\prime} L$ is the round-trip travel time through the $\mathrm{ZnSe}$ window. The first term in Eq. (13), $\Lambda_{\mathrm{F}}(\tau)$, which represents the individual QOCT scan of the front window, is unaffected by the presence of the dispersive $\mathrm{ZnSe}$ medium. The second term of Eq. (13), representing the QOCT scan of the back window, behaves as $|\alpha|^{2} \Lambda_{\mathrm{B}}$. The multiplication by the loss factor $|\alpha|^{2}$ simply results in a reduction of visibility. Neither $\beta_{0}$ nor the GVD parameter $\beta^{\prime \prime}$ appear in the second term; nor, in fact, would any higher even-order terms were we to carry the Taylor expansion further. Thus the features associated with the scan of the back window remain unaffected by dispersion.

The cancellation of GVD is an important signature of QOCT. In OCT, $\beta$ " does not cancel and the result is a degradation of depth resolution and a reduction of the signal-to-noise ratio [16].

On the other hand, the third term in Eq. (13), $\Lambda_{\mathrm{FB}}^{\text {disp }}$, which is a dispersed version of $\Lambda_{\mathrm{FB}}$, the third term appearing in Eq. (8), is given by

$$
\begin{aligned}
\Lambda_{\mathrm{FB}}^{\operatorname{disp}}(\tau)=2 \operatorname{Re}\left\{\alpha ^ { * } \left[r_{1} r_{3}^{*} s^{\operatorname{disp}}(\tau)\right.\right. & +\left(r_{1} r_{4}^{*} e^{-i \omega_{0} \tau_{d}}+r_{2} r_{3}^{*} e^{i \omega_{0} \tau_{d}}\right) s^{\operatorname{disp}}\left(\tau-\tau_{d}\right) \\
& \left.\left.+r_{2} r_{4}^{*} s^{\operatorname{disp}}\left(\tau-2 \tau_{d}\right)\right] e^{-i\left(\omega_{0} \tau_{d}+2 \beta_{0} L\right)}\right\},
\end{aligned}
$$

where $s^{\operatorname{disp}}(\tau)$ is the Fresnel transform

$$
s^{\operatorname{disp}}(\tau)=\int d \Omega S(\Omega) e^{-i 2 \beta^{\prime \prime} \Omega^{2} L} e^{-i \Omega \tau} .
$$

The sensitivity of the cross-window intermediate terms in $\Lambda_{\mathrm{FB}}^{\text {disp }}$ to the dispersiveness of the ZnSe medium permits the measurement of its GVD coefficient $\beta^{\prime \prime}$.

Figure 4(a) displays the normalized QOCT interferogram for this sample. As predicted by Eq. (13), the widths of all features in $\Lambda_{\mathrm{F}}$ and $\Lambda_{\mathrm{B}}$ remain essentially as they were in the absence of the dispersive medium [see Fig. 3(a)]. These features encompass all the dips labelled $(j, j)$, which carry the structural information of the sample. Thus the optical sectioning capability of the QOCT technique is unaffected by the presence of the dispersive medium.

There is a slight broadening from $18.5 \mu \mathrm{m}$ to $19.5 \mu \mathrm{m}$ of the three features in the leftmost cluster labelled $\Lambda_{\mathrm{B}}$. This could result from the sensitivity of QOCT to the higher odd-order terms in the Taylor expansion of $\beta\left(\omega_{0}+\Omega\right)$, namely $\beta^{\prime \prime \prime}, \beta^{V}, \ldots$ etc, which are not cancelled. In the case at hand, however, numerical simulation reveals that the broadening associated with $\beta^{\prime \prime \prime}(\approx 0.05 \mu \mathrm{m})$ is far less than that observed in the experiment. The broadening appears rather to be a consequence of the diffraction of the beam as it propagates and its subsequent passage through an aperture, which results in a truncation of the spectrum of the light, an effect we have previously observed in other experiments. The center features of the triplets, $(1,2)$ and $(3,4)$, 
in the QOCT interferogram displayed in Fig. 4(a), are also unaffected by the presence of the $\mathrm{ZnSe}$, since they are sensitive only to the dispersion of the fused silica, which is negligible. This would not be the case, however, if the interstitial materials between the reflecting surfaces, 1 and 2 (front window), and/or 3 and 4 (back window), were dispersive [7].

On the other hand, the intermediate features, labelled $(1,3),(1,4),(2,3)$, and $(2,4)$, appearing in the center cluster denoted $\Lambda_{\mathrm{FB}}^{\text {disp }}$ are broadened $(\mathrm{FWHM}=35.5 \mu \mathrm{m})$. This broadening arises from the dispersiveness of the ZnSe medium lying between the $(j, k)$ pair of reflecting surfaces appearing in their labels. Given the thickness of the $\mathrm{ZnSe}, L=12 \mathrm{~mm}$, and fitting the data to Eq. (15), the value of the extracted GVD coefficient $\beta^{\prime \prime}$ is determined to be $\beta^{\prime \prime} \approx 4.0 \pm$ $0.8 \times 10^{-25} \mathrm{~s}^{2} \mathrm{~m}^{-1}$. This result is in good agreement with the nominal value obtained using the Sellmeier equation, $\beta^{\prime \prime} \approx 5.4 \times 10^{-25} \mathrm{~s}^{2} \mathrm{~m}^{-1}$ [17], and with the value obtained via direct computation from $n(\lambda)$ (the refractive index as a function of wavelength [18]), which is $\beta^{\prime \prime} \approx$ $5.2 \times 10^{-25} \mathrm{~s}^{2} \mathrm{~m}^{-1}$.

In contrast, in the normalized OCT interferogram displayed in Fig. 4(b), the two interferencefringe envelopes associated with reflections from the surfaces of the back window are broadened from 37 to $104 \mu \mathrm{m}$ as a result of dispersion. Those of the front window are clearly unaffected by the dispersion of the medium that lies below the window.

\section{Discussion}

In the absence of prior information relating to the structure of the sample, features in the QOCT interferogram associated with reflections from a single surface (referred to as the "first class" of features) may be confounded with cross-interference features associated with pairs of surfaces (the "second class" of features). These two classes may be readily distinguished, however, since slight variations of the pump frequency change the form of features in the second class (e.g., from dips to humps), whereas those in the first class are invariant to such variations.

Thus, dithering the pump frequency, for example, can serve to wash out the features in the second class, thereby leaving intact the dispersion-cancelled portion of the interferogram that reveals the axial structure of the sample [7]. We expect that returns from scattering media would have this same effect because of the associated random phases. Simple subtraction of this pattern from the undithered version allows the second class of features to be separated, thereby also providing a dispersion-sensitive determination of the GVD coefficients of the various media that comprise the sample.

The signal-to-noise ratio (SNR) for the OCT and QOCT interferograms is determined by a number of factors, including the optical power in the interferometer, the transmittances of the optical paths, the quantum efficiency of the detector(s), and the duration of the experiment. These parameters play different roles in OCT and QOCT; in the case at hand, they varied from one experiment to another, as is clear in Figs. 3 and 4.

\section{Conclusion}

We have carried out experiments demonstrating dispersion-cancelled and dispersion-sensitive quantum optical coherence tomography (QOCT). The three principal advantages that stem from the frequency entanglement of the twin-photon source: dispersion cancellation, resolution doubling, and the ability to directly measure the GVD coefficient, have been illustrated.

\section{Acknowledgments}

This work was supported by the National Science Foundation; by the Center for Subsurface Sensing and Imaging Systems (CenSSIS), an NSF Engineering Research Center; and by the David \& Lucile Packard Foundation. 